\title{
A Continuous Renal Replacement Therapy Protocol for Patients with Acute Kidney Injury in Intensive Care Unit with COVID-19
}

\author{
Federico Nalesso ${ }^{1}$, Francesco Garzotto ${ }^{2}$, Leda Cattarin ${ }^{1}{ }^{1}$, Laura Gobbi ${ }^{1}$, Laila Qassim ${ }^{1}{ }^{10}$, \\ Luca Sgarabotto ${ }^{1}$ (1) , Ivo Tiberio ${ }^{3}$ and Lorenzo A. Calò ${ }^{1, *(1)}$ \\ 1 Department of Medicine, Nephrology, Dialysis and Transplantation Unit, University of Padova, \\ 35128 Padova, Italy; federico.nalesso@aopd.veneto.it (F.N.); leda.cattarin@gmail.com (L.C.); \\ laura.gobbi@hotmail.it (L.G.); laila.87@virgilio.it (L.Q.); sgarabottoluca@gmail.com (L.S.) \\ 2 Veneto Institute of Oncology IOV-IRCCS, Healthcare Directorate Unit, 35128 Padova, Italy; \\ f.garzotto@gmail.com \\ 3 Emergency-Urgency Department, Anesthesia and Intensive Care, Azienda Ospedaliera di Padova University \\ of Padova, 35128 Padova, Italy; ivo.tiberio@aopd.veneto.it \\ * Correspondence: renzcalo@unipd.it; Tel.: +39-049-8213071; Fax: +39-049-8217921
}

Received: 7 April 2020; Accepted: 18 May 2020; Published: 19 May 2020

\begin{abstract}
COVID-19 often leads to acute respiratory distress syndrome complicated by acute kidney injury (AKI). The indications for renal replacement therapy for these patients are those commonly accepted to treat AKI. We describe a continuous veno-venous haemodialysis (CVVHD) protocol for AKI, which aims to provide the best treatment according to the particular patient's and medical personnels' needs in biohazard settings with limited human and technological resources. We designed a CVVHD protocol with a high cut-off (HCO) filter in regional citrate anticoagulation (RCA). The HCO filter in diffusion determines the enhanced cytokines clearance with less filter clotting due to a lower filtration fraction. In our hospital, at the beginning of the pandemic outbreak, we treated seven COVID-19 patients with AKI stage 2 and 3 and recorded the circuit lifespan and the number of interventions on monitors. CVVHD in RCA appears to be safe, effective and easy to be performed in a biohazard scenario using lower blood flows and less bag changes with fluid savings, a biohazard reduction and sparing of resources. Although the data come from a very small cohort, our protocol seems related to a low mortality.
\end{abstract}

Keywords: Covid-19; AKI; ARDS; CRRT; CVVHD; HCO; cytokines; intensive care

\section{Introduction}

The SARS-CoV-2 infection, termed coronavirus disease 2019 (COVID-19), appeared for the first time in Italy, among the European countries, with two simultaneous outbreaks in the Lombardy and Veneto regions. Currently, COVID-19 cases are registered in all Italian regions and the epidemic is spreading through Europe. Our Veneto Regional Hub Hospital for COVID-19 in Padua, has firstly faced an increasing number of patients admitted to the intensive care unit (ICU) due to severe illness. Patients affected by COVID-19 present a dysfunctional immune system with an uncontrolled immune response, leading to a "cytokine storm" associated with a severe lung injury [1], as described for SARS-CoV, in which was described an increased proinflammatory cytokines level associated with pulmonary inflammation and extensive lung damage [2]. Patients affected by COVID-19 requiring ICU admission have higher levels of cytokines, suggesting that the "cytokine storm" is associated with the disease severity [3]. 
Acute kidney injury (AKI) is a common complication that occurs in $50-70 \%$ of ICU patients and is associated with worse outcome, with mortality rates of nearly $50 \%$ [4].

Conventional COVID-19 therapy typically starts with resuscitative measures in ICU [5,6]. While an effective therapy for COVID-19 does not exist yet, the indications for renal replacement therapy (RRT) should be those widely accepted to AKI treatment, restoring the immune homeostasis, removing inflammatory mediators causing acute respiratory distress syndrome (ARDS) and preventing and correcting the fluid balance when diuretics are not effective. Fluid overload, in fact, is a known independent risk factor for ICU mortality [7] and leads to several complications such as pulmonary edema, further worsening the respiratory system. A recent Acute Dialysis Quality Initiative conference has extensively addressed the topic of patient selection and timing for continuous renal replacement therapy (CRRT) [8]. Conversely, data on patients with COVID-19 are still discordant due to the large practice variation on the management of this disease $[9,10]$, mainly for the different local experience and resources. Moreover, in a biohazard setting, it is necessary to provide a simple and easy CRRT to determine the renal substitution and multiorgan support [11] by reducing the "cytokine storm" and control the fluid balance. In a biohazard scenario, it is necessary to improve technological and human resources by optimizing patient care and reducing the risk of medical staff contamination. CRRTs are characterized by high technological complexity and the need for supervision and maintenance can become critical in this particular ICU setting. The complexity of ARDS patients requires intensive care including the planned pronation from the supine position and vice versa more times per day. Moreover, the performance of central venous catheters (CVC) for extracorporeal circulation (EC) can be suboptimal, inducing reduced blood flows, as in the case for patients undergoing extracorporeal membrane oxygenation (ECMO) if the CRRT is performed using a CVC in a central vein. The blood flow in the EC, in fact, becomes critical as low flows correspond to a greater risk of circuit coagulation, less depurative clearances and greater filtration fractions (FF) in convective processes such as continuous veno-venous hemofiltration (CVVH) compared with diffusive processes such as continuous veno-venous hemodialysis (CVVHD). In addition, convective therapies generally require higher blood flows compared with the continuous veno-venous hemodialysis (CVVHD), resulting in recurrent alarms for an inadequate blood flow with consequent therapy downtimes and loss of a depurative dose. Regarding the purification characteristics necessary for ARDS patients with AKI, which includes the removal of cytokines, the replacement of the renal function and the maintenance of the fluid balance, we have set up an extracorporeal blood purification technique able to remove cytokines, replacing the kidney function using relatively lower blood flows and FF, ensuring the longest circuit lifespan with no therapy downtimes. Furthermore, we identified the best depurative technique requiring the lowest number of maintenance interventions for the fluid bags changing in order to reduce staff exposure to the COVID-19 patients and to potentially infected effluent fluids.

\section{Experimental Section}

At the very beginning of the outbreak in our region, we organized a team of intensivists, nephrologists and engineers aimed to define the most efficient strategy to provide a CRRT in the frame of limited human and technological resources as in a critical biohazard setting.

With the current knowledge on COVID-19 and according to our local resources, we designed a continuous veno-venous haemodialysis with a high cut-off membrane (HCO) in regional citrate anticoagulation (RCA) in the so-called RCA-HCO-CVVHD treatment. Heparin is also available for this treatment when RCA is contraindicated. No extra CRRT anticoagulants are used on concomitant ECMO patients. The use of a HCO filter in the diffusive modality with lower blood flows determines a reduced filtration fraction (FF), with a high clearance of small and medium molecules and an enhanced high molecular weight clearance for cytokines [12]. Our standard protocol for RCA-CVVHD requires a HCO filter (Septex, Baxter, IL, USA) with a blood flow $\left(\mathrm{Q}_{\mathrm{B}}\right)$ of $120-160 \mathrm{~mL} / \mathrm{min}$, a dialysate calcium free flow $\left(Q_{D}\right)$ of $30 \mathrm{~mL} / \mathrm{Kg} / \mathrm{h}$ and a $4 \%$ concentrated citrate solution $(136 \mathrm{mmol} / \mathrm{L})$ at the flow of $3.0 \mathrm{mmol} / \mathrm{L}$ balanced by calcium chloride infusion $(680 \mathrm{mmol} / \mathrm{L})$ in a patient's central vein according to a specific 
monitor algorithm (Prismaflex, Baxter, IL, USA) (Table 1). According to our protocol, systemic and post-filter ionized calcium (iCa) must be controlled after the beginning of the treatment at $30 \mathrm{~min}, 2 \mathrm{~h}$, $6 \mathrm{~h}$ and every $6 \mathrm{~h}$. In case of a citrate dose or calcium compensation variation, an extra control after $2 \mathrm{~h}$ is required. The total blood calcium (Сатот) concentration should be evaluated in order to calculate the $\mathrm{Ca}_{\mathrm{TOT}} / \mathrm{iCa}$ at least once daily to early identify a citrate accumulation detected by a ratio $>2.25$ [13]. The citrate dose and the calcium compensation are set depending on the serum calcium values at the planned checks according to their specific algorithms reported in Tables 2 and 3. Finally, although the collection of biological samples for the evaluation of patients' cytokine levels upon our treatment protocol would have been necessary, it was intentionally not done due to obvious safeness reasons given the patients' SARS-CoV-2 infection. However, based on the abovementioned characteristics of HCO used and supported by a large documentation regarding its effect on the reduction/clearance of cytokines, an effect on patients' cytokine levels is implied.

Table 1. Protocol parameters for the use of Septex with Prismaflex $\S$.

\begin{tabular}{|c|c|c|c|c|}
\hline $\begin{array}{l}\text { Treatment } \\
\text { Parameters }\end{array}$ & RCA-CVVHD & Heparin-CVVHD & $\begin{array}{l}\text { CVVHD without } \\
\text { Anticoagulation }\end{array}$ & $\begin{array}{l}\text { RCA-CVVHD: Treatment } \\
\text { for } 80 \mathrm{Kg} \text { Patient }\end{array}$ \\
\hline $\begin{array}{l}\text { Blood flow rate } \\
(\mathrm{mL} / \mathrm{min})\end{array}$ & $120-160$ & $160-200$ & $160-200$ & 140 \\
\hline $\begin{array}{l}\text { Dialysis dose } \\
(\mathrm{mL} / \mathrm{kg} / \mathrm{h})\end{array}$ & $30^{\mathrm{A}}$ & $30^{B, C}$ & $30^{B, C}$ & $30^{\text {A }}(2400 \mathrm{~mL} / \mathrm{h})$ \\
\hline $\begin{array}{l}4 \% \text { citrate flow } \\
(\mathrm{mL} / \mathrm{h})\end{array}$ & $160-210$ & - & - & 185 \\
\hline $\begin{array}{c}\text { Calcium } \\
\text { compensation } \\
(\%)\end{array}$ & $100^{*}$ & - & - & $100^{*}$ \\
\hline $\begin{array}{l}\text { Citrate dose } \\
(\mathrm{mmol} / \mathrm{L})\end{array}$ & $3.0^{\#}$ & - & - & $3.0^{\#}$ \\
\hline
\end{tabular}

Table 2. Algorithm for the citrate dose management.

\begin{tabular}{cccc}
\hline Treatment Parameters & & & \\
\hline & LOW & NORMAL & HIGH \\
\hline $\begin{array}{c}\text { Post-filter ionized } \\
\text { calcium }(\mathrm{mmol} / \mathrm{L})\end{array}$ & $<0.25$ & $0.25-0.50$ & $>0.50$ \\
\hline Citrate dose $(\mathrm{mmol} / \mathrm{L})$ & $2.7 \mathrm{mmol} / \mathrm{L}$ & $3.0 \mathrm{mmol} / \mathrm{L}$ & $3.3 \mathrm{mmol} / \mathrm{L}$ \\
\hline
\end{tabular}

Table 3. Algorithm for the calcium compensation management.

\begin{tabular}{cccccc}
\hline Treatment Parameters & & & & & \\
\hline Systemic ionized calcium $(\mathrm{mmol} / \mathrm{L})$ & $<0.8$ & $0.8-0.9$ & $1.0-1.2$ & $1.2-1.35$ & $>1.35$ \\
\hline Calcium compensation & $+30 \%$ & $+20 \%$ & - & $-10 \%$ & $-20 \%$ \\
\hline
\end{tabular}

In case of an ECMO patient, or if citrate and heparin are contraindicated, the $\mathrm{Q}_{\mathrm{B}}$ can be increased to $160-200 \mathrm{~mL} / \mathrm{min}$ to reduce the circuit's coagulation, maintaining the same $\mathrm{Q}_{\mathrm{D}}$ with a standard dialysate solution selected according to a patient's ions and bicarbonate levels (Table 1). In order to customize the treatment and reduce the need for electrolyte replacement (which would increase the nurse's workload), solutions with different concentrations of potassium ( 2 and $4 \mathrm{mmol} / \mathrm{L}$ ), magnesium 
( 0.5 and $0.6 \mathrm{mmol} / \mathrm{L})$ and phosphorus $(0$ and $1.2 \mathrm{mmol} / \mathrm{L})$ allow a flexible electrolytes management in all patients treated with heparin or without anticoagulation.

To achieve a daily depurative dose of $25 \mathrm{~mL} / \mathrm{kg} / \mathrm{h}$ according to KDIGO, a higher value, usually ranging from 25 to $30 \mathrm{~mL} / \mathrm{Kg} / \mathrm{h}$, needs to be prescribed considering the potential downtimes due to the alarms and the bag changes. Indeed, we calculated a mean time from the empty bag alarm to the complete resolution of $15 \mathrm{~min}$ for any bag change due to the need for personal protective equipment (PPE) in this biohazard contest in a COVID-19 ICU. In the protocol, the depurative dose of $30 \mathrm{~mL} / \mathrm{kg} / \mathrm{h}$ is estimated to be suitable for compensating the downtimes of our clinical reality.

\section{Results}

Seven patients were treated with the RCA-HCO-CVVHD protocol until now (Table 4). The mean age was $68.1 \pm 6.77$ years, most male $(85.7 \%$ ) and overweight (mean weight was $91.0 \pm 11.34 \mathrm{~kg}$ ). At baseline, $85.7 \%$ had hypertension, $57.1 \%$ has a pre-existing chronic kidney disease and $42.9 \%$ had diabetes mellitus.

Table 4. Patients and treatments characteristics.

\begin{tabular}{cc}
\hline \multicolumn{2}{c}{ Patients Characteristics at Baseline $(\boldsymbol{n}=\mathbf{7})$} \\
\hline Age (years), Mean \pm SD & $68.1 \pm 6.77$ \\
Male, $n(\%)$ & $6(85.7 \%)$ \\
Chronic kidney disease, $n(\%)$ & $4(57.1 \%)$ \\
Diabetes mellitus, $n(\%)$ & $3(42.9 \%)$ \\
Arterial hypertension, $n(\%)$ & $6(85.7 \%)$ \\
Weight (Kg), mean \pm SD & $91.0 \pm 11.34$ \\
AKI stage 2, $n(\%)$ & $6(85.7 \%)$ \\
AKI stage 3, $n(\%)$ & $1(14.3 \%)$ \\
C reactive protein (mg/L), mean \pm SD & $153.28 \pm 118.61$ \\
Treatment Characteristics (RCA-HCO-CVVHDF) \\
Blood flow rate (mL/min), mean \pm SD & $144.3 \pm 11.34$ \\
Dialysis dose (mL/h), mean \pm SD & $2757.1 \pm 320.71$ \\
Number of circuits for patients, mean \pm SD & $2.71 \pm 0.76$ \\
Hours of treatment for patients, mean \pm SD & $195.43 \pm 5.43$ \\
\hline
\end{tabular}

The application of our protocol in all seven patients allowed to reach $72 \mathrm{~h}$ of treatment for all 19 circuits used (mean circuits for patients $2.71 \pm 0.76$ ), without technical downtimes due to a central venous catheter malfunction or circuit/filter coagulation. Over $1300 \mathrm{~h}$ of CVVHD therapy (mean hours per patient $195.43 \pm 5.43$ ) were guaranteed, interrupted only by the bags change and scheduled circuit reset on expiry. The mean blood flow rate was $144.3 \pm 11.34 \mathrm{~mL} / \mathrm{min}$ and the mean dialysis dose was $2757.1 \pm 320.71 \mathrm{~mL} / \mathrm{h}$, both calculated per total time. The patients' prone ventilation did not cause changes in the blood flows inducing alarms or discontinuations of the treatment due to the reduced blood flows set. The extracorporeal circulation stability has considerably reduced the nurses' interventions, decreasing the risk of contamination. No staff biohazard contamination due to CVVHD patient management was experienced. As a precaution, the effluent disposal was collected through a biohazard bin and not through the standard drainage to avoid further contact with potentially contaminated fluid.

The systemic calcium compensation algorithm of the CRRT monitor has shown to maintain the serum calcium in the physiological range in all patients. No patient had signs of citrate toxicity.

All patients obtained a CRRT steady state in the control of ions, hydration status and acid base balance with urea and creatinine in the physiological range. No episodes of metabolic acidosis or alkalosis were reported during the CRRT treatments.

Although the data come from a very small cohort, our protocol seems related to a low mortality rate (two patients of seven). 


\section{Discussion}

Although convective therapies $(\mathrm{CVVH})$ are technically the most indicated modality for the removal of large molecules such as cytokine and myoglobin, the disadvantage of pre- or post-dilution on the circuit management limits their use in a biohazard setting, where the bleeding risk, the request for low effluent volumes and minimal technical interventions on the CRRT monitors are essential for the biocontainment. In particular in $\mathrm{CVVH}$, pre-infusion decreases the depurative efficacy due to the blood dilution, while the post-infusion promotes filter clotting due to the increased FF and filter hemoconcentration [14] promoting a premature circuit and blood losses, and higher workloads and costs-resources. In addition, the increased blood flows required in $\mathrm{CVVH}$ can result in a greater number of alarms for CVC malfunction with a consequent need for technical interventions by personnel. This element is important in relation to the patients' pronation as potential CVC dysfunctions can occur at a higher blood flow.

The advantages of RCA-HCO-CVVHD compared with the standard CVVH modality deal with a minor effluent volume, fewer bag interventions, a lower FF with a higher filter and circuit lifespan (also in the event of contraindications for the heparin use) and less alarm for complications related to the $\mathrm{Q}_{\mathrm{B}}$ due to a CVC malfunction.

For an $80 \mathrm{~kg}$ patient without net fluid removal and a $Q_{B}$ of $140 \mathrm{~mL} / \mathrm{min}$ with a hematocrit of 0.30, the pre-infusion in the CVVH modality has to be set to $3200 \mathrm{~mL} / \mathrm{h}$, considering the dilution factor, compared with $2200 \mathrm{~mL} / \mathrm{h}$ of dialysate in CVVHD to achieve the same depurative dose of $25-30 \mathrm{~mL} / \mathrm{kg} / \mathrm{h}$. This dose in the CVVHD modality allows personnel to provide 11 bag changes (5000 mL bag) compared with 16 in CVVH. Four fewer interventions in CVVHD mean a gain of $60 \mathrm{~min}$ nurse time per treatment per day, a saving of $20 \mathrm{~L}$ (four bags) per treatment per day and four PPE kits, as well as a decreased risk of a contamination. All these factors are very important in a scenario of reduced human and medical supplies.

The use of RCA-HCO-CVVHD therefore allows an effective renal purification in patients with ARDS and AKI in ICUs in a scenario of reduced human resources. Furthermore, although we have not measured patients' cytokine levels upon our treatment protocol, in addition to the large available documentation regarding cytokine removal by the HCO used in our protocol [12], the recovery of 5 out of 7 patients from respiratory failure, the end of their need of invasive mechanical ventilation and even their discharge from the ICU are important indirect clinical proofs to be considered. These clinical characteristics, which followed our treatments, in fact, make highly likely the impact of our treatments on cytokines, by reducing/clearing their levels.

The RCA-HCO-CVVHD advantages compared with an equivalent dose in CVVH are a lower effluent volume, fewer bag interventions, lower FF with a higher filter and circuit lifespan and less alarms for complications related to the $\mathrm{Q}_{\mathrm{B}}$ due to a CVC malfunction. The possibility of performing the treatment with lower blood flows has resulted in greater stability of the extracorporeal circulation and fewer alarms with reduced downtimes. This stability, obtained with the reduced blood flows used in the protocol, has been maintained also during and after the patient's pronation and supination maneuvers. Moreover, the absence of coagulation of the extracorporeal circulation obtained by RCA has determined the achievement of $72 \mathrm{~h}$ of treatment for all the circuits used, decreasing the nurses' interventions. The absence of circuit clotting avoided significant blood losses requiring blood transfusion. Moreover, the reduction of the technical interventions on the CRRT monitors due to the greater stability of the extracorporeal circulation and the absence of treatment interruptions for circuit coagulation have limited the nurses' contacts with the patient only to the bag changes. This has reduced the workload and the number of personal protective equipment used for the CRRT management, saving resources. In our experience, no nurses were contaminated during the procedures related to the CRRT administration and management. 


\section{Conclusions}

From the data obtained until now, the RCA-HCO-CVVHD appears to be safe, effective and easy to apply in a biohazard scenario with limited human resources. Further clinical application of this protocol will allow the collection of more data to confirm the clinical effectiveness of RCA-HCO-CVVHD.

Finally, depending on the desired purification strategy, the local skills and the availability, alternatives treatment can include [11] the use of a high molecular weight filter such as an EMIC2 (Fresenius Medical Care, Bad Homburg Germany), an adsorptive hemofiltration filter such as oXiris (Baxter, IL, USA), the adsorption characteristics of the Hemofeel (Toray Medical Co. Ltd., Tokyo, Japan) in CVVHD or the use of sorbents such as polymyxin B (Toray Medical Co. Ltd., Tokyo, Japan), CytoSorb (CytoSorbents Europe GmbH, Berlin, Germany), HA-330 (Jafron, China) and the Alteco LPS adsorber (Alteco Medical AB, Lund, Sweden).

Author Contributions: F.N.: Conceptualization of the study, methodology, validation and original draft writing; F.G. contributed to validation and original draft writing; L.A.C. contributed to original draft writing and reviewed the manuscript; I.T. reviewed the manuscript and followed patients; L.C. contributed to original draft writing and followed the patients; L.G., L.Q., L.S. collected and cured the data and followed patients. All authors have read and agreed to the published version of the manuscript.

Funding: This research received no external funding.

Acknowledgments: The authors thank all the nurses who participated in the management of CRRT treatments.

Conflicts of Interest: The authors declare no conflict of interest.

\section{References}

1. Wong, C.K.; Lam, C.W.; Wu, A.K.; Ip, W.K.; Lee, N.L.; Chan, I.H.; Lit, L.C.; Hui, D.S.; Chan, M.H.; Chung, S.S.; et al. Plasma inflammatory cytokines and chemokines in severe acute respiratory syndrome. Clin. Exp. Immunol. 2004, 136, 95-103. [CrossRef] [PubMed]

2. Mahallawi, W.H.; Khabour, O.F.; Zhang, Q.; Makhdoum, H.M.; Suliman, B.A. MERS-CoV infection in humans is associated with a pro-inflammatory Th1 and Th17 cytokine profile. Cytokine 2018, 104, 8-13. [CrossRef] [PubMed]

3. Huang, C.; Wang, Y.; Li, X.; Ren, L.; Zhao, J.; Hu, Y.; Zhang, L.; Fan, G.; Xu, J.; Gu, X.; et al. Clinical features of patients infected with 2019 novel coronavirus in Wuhan, China. Lancet 2020, 395, 497-506. [CrossRef]

4. Griffin, B.R.; Liu, K.D.; Teixeira, J.P. Critical Care Nephrology: Core Curriculum 2020. Am. J. Kidney Dis. 2020, 75, 435-452. [CrossRef] [PubMed]

5. Rhodes, A.; Evans, L.E.; Alhazzani, W.; Levy, M.M.; Antonelli, M.; Ferrer, R.; Kumar, A.; Sevransky, J.E.; Sprung, C.L.; Nunnally, M.E.; et al. Surviving Sepsis Campaign: International Guidelines for Management of Sepsis and Septic Shock: 2016. Intensive Care Med. 2017, 43, 304-377. [CrossRef] [PubMed]

6. WHO. Clinical Management of Severe Acute Respiratory Infection When Novel Coronavirus (2019-nCoV) Infection is Suspected Interim Guidance. Available online: https:/www.who.int/docs/default-source/ coronaviruse/clinical-management-of-novel-cov.pdf (accessed on 4 March 2020).

7. Garzotto, F.; Ostermann, M.; Martin-Langerwerf, D.; Sanchez-Sanchez, M.; Teng, J.; Robert, R.; Marinho, A.; Herrera-Gutierrez, M.E.; Mao, H.J.; Benavente, D.; et al. The Dose Response Multicentre Investigation on Fluid Assessment (DoReMIFA) in critically ill patients. Crit. Care 2016, 20, 196. [CrossRef] [PubMed]

8. Ostermann, M.; Joannidis, M.; Pani, A.; Floris, M.; De Rosa, S.; Kellum, J.A.; Ronco, C.; 17th Acute Disease Quality Initiative Consensus G. Patient Selection and Timing of Continuous Renal Replacement Therapy. Blood Purif. 2016, 42, 224-237. [CrossRef] [PubMed]

9. Bouadma, L.; Lescure, F.X.; Lucet, J.C.; Yazdanpanah, Y.; Timsit, J.F. Severe SARS-CoV-2 infections: Practical considerations and management strategy for intensivists. Intensive Care Med. 2020. [CrossRef] [PubMed]

10. Chen, N.; Zhou, M.; Dong, X.; Qu, J.; Gong, F.; Han, Y.; Qiu, Y.; Wang, J.; Liu, Y.; Wei, Y.; et al. Epidemiological and clinical characteristics of 99 cases of 2019 novel coronavirus pneumonia in Wuhan, China: A descriptive study. Lancet 2020, 395, 507-513. [CrossRef] 
11. Ankawi, G.; Neri, M.; Zhang, J.; Breglia, A.; Ricci, Z.; Ronco, C. Extracorporeal techniques for the treatment of critically ill patients with sepsis beyond conventional blood purification therapy: The promises and the pitfalls. Crit. Care 2018, 22, 262. [CrossRef] [PubMed]

12. Villa, G.; Zaragoza, J.J.; Sharma, A.; Neri, M.; De Gaudio, A.R.; Ronco, C. Cytokine removal with high cut-off membrane: Review of literature. Blood Purif. 2014, 38, 167-173. [CrossRef] [PubMed]

13. Meier-Kriesche, H.U.; Gitomer, J.; Finkel, K.; DuBose, T. Increased total to ionized calcium ratio during continuous venovenous hemodialysis with regional citrate anticoagulation. Crit. Care Med. 2001, 29, 748-752. [CrossRef] [PubMed]

14. Joannidis, M.; Oudemans-van Straaten, H.M. Clinical review: Patency of the circuit in continuous renal replacement therapy. Crit. Care 2007, 11, 218. [CrossRef] [PubMed]

(C) 2020 by the authors. Licensee MDPI, Basel, Switzerland. This article is an open access article distributed under the terms and conditions of the Creative Commons Attribution (CC BY) license (http://creativecommons.org/licenses/by/4.0/). 